\title{
长江口沿岸碎波带仔稚鱼的种类组成及其多样性特征
}

\author{
蒋日进 ${ }^{1}$, 钟俊生 ${ }^{1, *}$, 张冬良 ${ }^{1}$, 傅萃长 ${ }^{2}$ \\ （1. 上海海洋大学 生命科学与技术学院, 上海 200090； 2. 复旦大学 生物多样性和生态工程教育部重点实验室, 上海 200433)
}

摘要: 为了进一步探明长江口沿岸碎波带仔稚鱼的种类组成和多样性特征, 2006 年 7 月-2007 年 6 月的每月 大潮期间, 在长江口沿岸碎波带 13 个站位点的水深 0.5-1.5 m 处, 两人沿海岸平行方向步行拖曳小型拖网 (1 m $\times 4$ $\mathrm{m}$, 网目 $1 \mathrm{~mm}$ ) 采集仔稚鱼样本。周年共拖网 397 网次, 采集到仔稚鱼 49045 尾。仔稚鱼隶属于 31 科, 共 84 种, 其中海洋性鱼类 16 种, 河口性鱼类 30 种, 洄游性鱼类 4 种, 淡水性鱼类 34 种。洄游性鱼类刀鲚占总个体 数的 $82.63 \%$, 为最优势种。仔稚鱼的物种数、个体数和丰度在冬季较低, 以 3 月份最低, 夏季最高。 Margalef 丰富度指数 2006 年 11 月-2007 年 4 月相对较低, 2006 年 10 月最高。Shannon-Wiener 多样性指数在 10 月最高, 7 月最低。Pielou 均匀度指数全年较恒定，2006 年 7 月和 2007 年 6 月相对较低。

关键词：长江口；碎波带；仔稚鱼；种类组成；多样性；季节动态

中图分类号：Q958.1 文献标识码：A＼cjkstart文章编号：0254-5853-(2008)03-0297-08

\section{Species Composition and Diversity of Fish Larvae and Juveniles in the Surf Zone of the Yangtze River Estuary}

\author{
JIANG Ri-jin ${ }^{1}$, ZHONG Jun-sheng ${ }^{1, *}$, ZHANG Dong-liang ${ }^{1}$, FU Cui-zhang ${ }^{2}$ \\ (1. College of Aqua-life Science and Technology, Shanghai Ocean University, Shanghai 200090, China; \\ 2. Ministry of Education Key Laboratory for Biodiversity Science and Ecological Engineering,Institute of Biodiversity Science, \\ School of Life Sciences, Fudan University, Shanghai 200433, China)
}

\begin{abstract}
This study investigated species composition and diversity of fish larvae and juveniles in the surf zone of the Yangtze River estuary to reveal the importance of this habitat as a fish nursery ground. Fish larvae and juveniles were collected monthly at 13 stations on the spring tide during July 2006 and June 2007. Samples were conducted along the surf zone (depth of $0.5-1.5 \mathrm{~m})$ using a seine net $(1 \mathrm{~m} \times 4 \mathrm{~m}, 1 \mathrm{~mm}$ mesh-aperture). Each haul was made parallel to the shore for a distance of about $50 \mathrm{~m}$. A total of 49045 fish larvae and juveniles were collected representing 84 species from 31 families. They included 16 marine species, 30 estuarine species, 4 diadromous species and 34 freshwater species. Coilia nasus was the most dominant species accounting for $82.63 \%$ of the total. The number of species, individual and abundance were lower in winter with the minimum number in March and maximum in summer. The Margalef index was relatively low between November 2006 and April 2007, the highest being in October 2006. The Shannon-Wiener diversity index was the highest in October 2006 and the lowest in March 2007. The Pielou evenness index was invariable throughout the whole year and relatively low in July 2006 and June 2007.
\end{abstract}

Key words: Yangtze River estuary; Surf zone; Fish larvae and juveniles; Species composition; Species diversity; Seasonal dynamics

碎波带是波浪在海岸附近发生破碎的区带 (Tang, 1983)。受地表径流、潮汐以及人类的活动 等的交互影响，碎波带水域的生态环境错综复杂。 Lasiak (1981) 首次研究了南非沿海碎波带的稚鱼
群落结构, Ruple（1984）提出了墨西哥湾沿岸碎波 带作为仔鱼栖息地的重要性。近年来, 日本、澳大 利亚、欧洲等地区的学者已开展了沿海碎波带仔稚 鱼的种类组成及其时空分布的大量研究, 证实了许

收稿日期：2007-12-17；接受日期：2008-03-19

基金项目：国家自然科学基金（30770364）；上海市教委一般项目（6680105221）; 农业部东海区渔政渔港监督管理局项目（6660106237); 上海市重点学科建设项目 (Y1101) 资助

*通讯作者 (Corresponding author), E-mail: jszhong@shou.edu.cn

第一作者简介: 蒋日进, 男, 硕士研究生, 研究方向为鱼类生态学。E-mail: jiangrijin@yahoo.com.cn 
多经济鱼类仔稚鱼均利用碎波带作为其保育场

(Senta \& Kinoshita, 1985; Kinoshita, 1993; Doherty, 1996; Bregje, 2001; Fujita et al, 2002; Zhong, 2006)。

在长江口及其邻近海域, 尽管大量的研究也涉 及了鱼类的群落结构（Wang et al, 1983; Tang, et al, 2003; Li et al, 2004, 2006; Liu et al, 2005; Zhang et al, 2007 ), 但对鱼卵、仔稚鱼的生态研究却相对较少, 而且研究水域主要集中在长江口门及其近海 $(\mathrm{Wu}$, 1989; Yang et al, 1990; Huang et al, 1992; Xu et al, 1999; Zhu et al, 2002; Shan et al, 2005; Jiang et al, 2006; Jin et al, 2007), 至今对于碎波带仔稚鱼研究 也仅见于 Zhong et al（2005, 2006, 2007）对其种类 组成的初步报道。为了进一步探明长江口沿岸碎波 带仔稚鱼类的种类组成, 本研究在长江口沿岸设置 的 13 个站位点的碎波带, 按月进行了仔稚鱼的周 年调查, 旨在充分展示长江口沿岸碎波带仔稚鱼的 多样性特征, 探讨碎波带作为其保育场的重要性, 提出仔稚鱼保护的措施, 为长江口渔业资源的可持 续利用提供科学依据。

\section{1 材料与方法}

\section{1 调查时间、地点及方法}

在长江口沿岸碎波带设置 13 个站位点（图 1), 2006 年 7 月 -2007 年 6 月, 每月大潮期间, 在水 深 0.5-1.5 $\mathrm{m}$ 处, 两人沿海岸平行方向, 步行拖曳 小型拖网 $(1 \mathrm{~m} \times 4 \mathrm{~m}$, 网目 $1 \mathrm{~mm})$, 拖曳距离约 $50 \mathrm{~m}$, 并记录拖曳的时间, 每个站位点重复拖网 2-3 次。 在现场用 $5 \%$ 的海水福尔马林固定标本, 并在现场 测量水温和盐度。

\section{2 室内工作}

将采集到的仔稚鱼鉴定到科、属、种，计数并 计算单位捕捞努力渔获量 (CPUE) (尾数/网次)。 按 Kendall (1984) 的仔稚鱼发育分期标准划分发育 阶段, 并用带有目测微尺的 Olympus SZ 解剖镜或 游标卡尺测取所有仔稚鱼的体长 (BL)。学名按照 Wu et al（1999）, 同属种名按英文字母进行排序。

\section{3 数据分析}

采用 Margalef 丰富度指数 $(D) 、$ Shannon-Wiener 多样性指数 $\left(H^{\prime}\right)$ 和 Pielou 均匀度指数 $\left(J^{\prime}\right)$ 进行 多样性研究 (Ma, 1994)。

Margalef 丰富度指数: $D=(S-1) / \ln N$

Shannon-Wiener 多样性指数: $H^{\prime}=-\sum P i \ln P i$

Pielou 均匀度指数: $J^{\prime}=H^{\prime} / \ln S$
式中, $S$ 为一个取样中的物种总数, $N$ 为一个取样 中总尾数, $P i$ 为 $i$ 种所占总尾数的比例。

以上统计分析过程均用 PRIMER 5.0 软件完成。

\section{2 结 果}

\section{1 温度与盐度的变化}

长江口沿岸碎波带年平均水温 $20.2^{\circ} \mathrm{C}, 8$ 月份 最高 $\left(35.0^{\circ} \mathrm{C}\right.$ )，2 月最低 $\left(4.0^{\circ} \mathrm{C}\right.$ )（图 2)。位于南 汇浅滩的 Sts. $1-3$ 和北支的 Sts. 10-13 受潮汐的影 响较大, 平均盐度较高, 其中 St.13 的平均盐度高 达 26.8; 而南支的 Sts.4-9 则受长江径流量的影响, 平均盐度一直处于低水平（2.8）（图 3)。

\section{2 种类组成}

周年调查共拖网 397 网次，采集到仔稚鱼 49 045 尾。仔稚鱼隶属于 31 科, 共 84 种。其中海洋 性鱼类 16 种, 河口性鱼类 30 种, 洄游性鱼类 4 种, 淡水性鱼类 34 种。洄游性鱼类刀鲚（Coilia nasus） 占总个体数的 $82.63 \%$, 为最优势种。其后依次为普

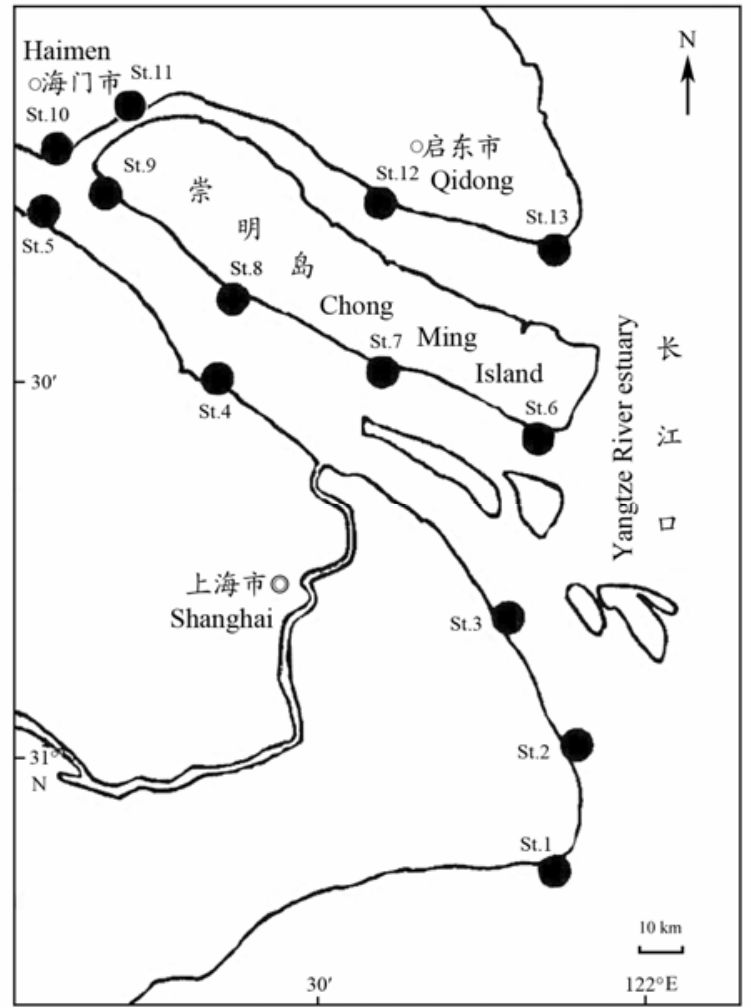

图 1 长江口沿岸碎波带仔稚鱼的调查站点

Fig. 1 Sampling stations for collecting fish larvae and juveniles in the surf zone of the Yangtze River estuary 




图 2 长江口沿岸碎波带水温的月变化

Fig. 2 Monthly water temperature in the surf zone of the Yangtze River estuary. Error bars represent S.D of the mean temperature among the 13 sampling stations in the same month.

氏细棘虾虎鱼（Acentrogobius pflaumi）（3.12\%）、 银飘鱼 (Pseudolaubuca sinensis) $(1.78 \%$ )、䱗 ( Hemiculter leucisculus) ( $1.53 \%$ )、弹涂鱼 ( Periophthalmus modestus) ( $1.34 \%$ )、似鱎 (Toxabramis swinhonis) (1.34\%)、纹缟虾虎鱼 (Tridentiger trigonocephalus) $(1.28 \%$ )、寡鳞飘鱼 (Pseudolaubuca engraulis) (1.07\%)、太湖新银鱼 (Neosalanx taihuensis) $(0.66 \%)$ 、鲻 ( Mugil cephalus $)(0.64 \%)$ 。以上 10 种的个体数占据了总 渔获个体数的 $95.39 \%$ （表 1 )。

最优势种刀鲚在 2006 年 7-10 月和 2007 年 6 月季节性出现在碎波带水域。其中在 6 和 7 月占绝 对优势, 分别为当月采集到仔稚鱼类的 $90.44 \%$ 和 $79.37 \%, 8$ 和 9 月相对较少, 10 月最少, 仅采集到 2 尾。

在长江口的沿海水域 (Sts.1-3, St.6, Sts.1213）周年共出现海洋性鱼类 15 种, 河口性鱼类 27 种, 洄游性 4 种, 淡水性鱼类 26 种。在长江口北 支的上游及南支淡水水域周年共出现海洋性鱼类 5 种, 河口性鱼类 20 种, 洄游性鱼类 2 种, 淡水性 鱼类 31 种。

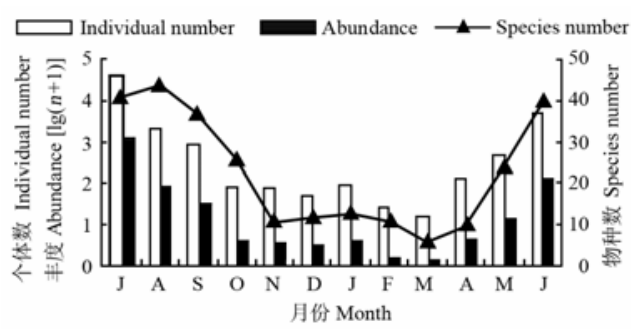

图 4 长江口沿岸碎波带仔稚鱼的物种数、个体数和 丰度的月变化

Fig. 4 Monthly changes of species number, individual number and abundance

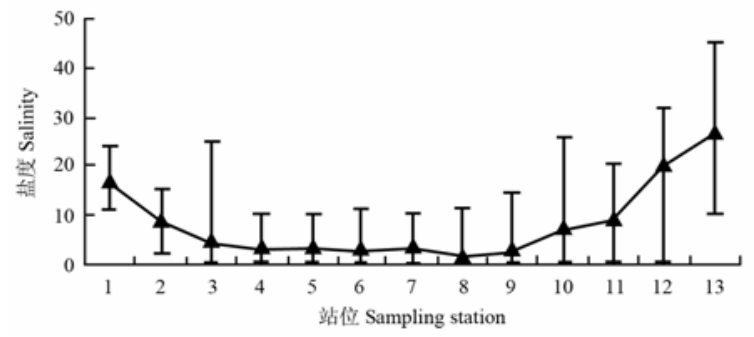

图 3 长江口沿岸碎波带各站位的盐度变化

Fig. 3 Mean salinity of each sampling station in the surf zone of the Yangtze River estuary. Error bars represent the ranges of the salinity in each station during the sampling period.

\section{3 仔稚鱼物种数、个体数和丰度的月变化}

从 2006 年 7 月 -2007 年 3 月, 仔稚鱼的物种 数、个体数和丰度的月变化呈递减趋势，2007 年 3 月至 6 月则呈递增趋势。其中 2006 年 7 月的个体 数最多, 为 39706 尾, 8 月的物种数最多, 为 44 种; 而 2007 年 3 月的仔稚鱼的物种数、个体数和 丰度均最低, 分别为 6 种、14 尾和 0.13 尾/网（图 4)。

\section{4 仔稚鱼的多样性指数的月变化}

碎波带仔稚鱼类的 Margalef 丰富度指数 $(D)$ 在 2006 年 11 月-2007 年 4 月较低, 其余月份相对 较高。其中 10 月最高 (5.76), 4 月最低 (1.85)。 Shannon-Wiener 多样性指数 $\left(H^{\prime}\right)$ 在 2006 年 10 月 最高 (2.78)，7 月最低 (0.56)。Pielou 均匀度指数 $\left(J^{\prime}\right)$ 月变化较恒定。由于刀鲚在碎波带的大量出 现, 2006 年 7 月和 2007 年 6 月的 $H^{\prime}$ 和 $J$ 值均相对 较低 (图 5)。

\section{3 讨 论}

\section{1 长江口碎波带的保育功能}

长江口作为我国最大的河口，渔业资源丰富，



图 5 长江口沿岸碎波带仔稚鱼的多样性指数的月变化 Fig. 5 Monthly changes of the diversity indexes in fish larvae and juveniles 
表 12006 年 7 月 -2007 年 6 月长江口沿岸碎波带采集的仔稚鱼种类组成

Tab. 1 Species composition of fish larvae and juveniles collected from the surf zone of Yangtze River estuary during July 2006 and June 2007

\begin{tabular}{|c|c|c|c|c|c|}
\hline 种名 Species & $\begin{array}{l}\text { 百分比 } \\
\text { Percentage in } \\
\text { numbers (\%) }\end{array}$ & $\begin{array}{l}\text { 体长范围 } \\
\text { Range of body } \\
\text { length }(\mathrm{mm})\end{array}$ & $\begin{array}{l}\text { 发育阶段 } \\
\text { Developmental } \\
\text { stage }\end{array}$ & $\begin{array}{l}\text { 生态类型 } \\
\text { Ecological } \\
\text { pattern }\end{array}$ & $\begin{array}{l}\text { 出现月份 } \\
\text { Month }\end{array}$ \\
\hline \multicolumn{6}{|l|}{ 海鲢科 Elopidae } \\
\hline 海鲢 Elops saurus & 0.10 & $10.8-33.9$ & $\mathrm{C}$ & $\mathrm{Ma}$ & $7-10,6$ \\
\hline \multicolumn{6}{|l|}{ 大海鲢科 Megalopidae } \\
\hline 大海鲢 Megalops cyprinoides & 0.31 & $16.1-27.8$ & $\mathrm{C}$ & $\mathrm{Ma}$ & $7-10$ \\
\hline \multicolumn{6}{|l|}{ 遮目鱼科 Chanidae } \\
\hline 遮目鱼 Chanos chanos & + & 12.2 & $\mathrm{C}$ & Ma & 8 \\
\hline \multicolumn{6}{|l|}{ 鲱科 Clupeidae } \\
\hline 鳓 Ilisha elongata & 0.02 & $3.0-10.4$ & A & Ma & 8,6 \\
\hline 斑䱕 Konosirus punctatus & + & 13.1 & $\mathrm{C}$ & Es & 6 \\
\hline \multicolumn{6}{|l|}{ 鳀科 Engraulidae } \\
\hline 刀鲚 Coilia nasus & 82.63 & $3.9-51.0$ & $\mathrm{~A}-\mathrm{D}$ & Di & $7-10,6$ \\
\hline 日本鳀 Engraulis japonicus & + & $8.7-13.1$ & $\mathrm{C}$ & $\mathrm{Ma}$ & 8,9 \\
\hline 斜领棱鳀 Thrissa purava & + & 13.4 & $\mathrm{C}$ & Ma & 8 \\
\hline \multicolumn{6}{|l|}{ 银鱼科 Salangidae } \\
\hline 寡齿新银鱼 Neosalanx oligodontis & 0.05 & $17.3-41.7$ & $\mathrm{C}-\mathrm{D}$ & $\mathrm{Fr}$ & $7,12,1,3,4$ \\
\hline 太湖新银鱼 Neosalanx taihuensis & 0.66 & $4.6-54.0$ & $\mathrm{~A}-\mathrm{D}$ & $\mathrm{Fr}$ & $7-12,1-6$ \\
\hline 有明银鱼 Salanx ariakensis & + & 128.4 & $\mathrm{D}$ & Es & 9 \\
\hline 银鱼科 Salangidae sp. & + & 13.6 & $\mathrm{~B}$ & $\mathrm{Fr}$ & 11 \\
\hline \multicolumn{6}{|l|}{ 鳗鲡科 Anguillidae } \\
\hline 日本鳗鲡 Anguilla japonica & + & 146.5 & $\mathrm{D}$ & Di & 10 \\
\hline \multicolumn{6}{|l|}{ 鲤科 Cyprinidae } \\
\hline 斑条鱊 Acheilognathus taenianalis & 0.01 & $8.6-47.7$ & $\mathrm{C}-\mathrm{D}$ & $\mathrm{Fr}$ & $7,2,6$ \\
\hline 鲫 Carassius auratus & 0.20 & $5.6-45.4$ & $\mathrm{C}-\mathrm{D}$ & $\mathrm{Fr}$ & $7-9,5,6$ \\
\hline 草鱼 Ctenopharyngodon idellus & 0.01 & $22.4-39.9$ & $\mathrm{D}$ & $\mathrm{Fr}$ & $7-9$ \\
\hline 红鳍鲌 Culter erythropterus & 0.39 & $8.6-84.5$ & $\mathrm{C}-\mathrm{E}$ & $\mathrm{Fr}$ & $7-11,2,5$ \\
\hline 蒙古红鲌 Erythroculter mongolicus & 0.03 & $28.2-41.8$ & $\mathrm{C}-\mathrm{D}$ & $\mathrm{Fr}$ & $7,9,11,12,1,2$ \\
\hline 油䱗Hemiculter bleekeri bleekeri & 0.03 & $38.0-80.0$ & $\mathrm{D}-\mathrm{E}$ & $\mathrm{Fr}$ & $8-10,12,1,2$ \\
\hline 餐Hemiculter leucisculus leucisculus & 1.53 & $6.0-73.6$ & $\mathrm{~B}-\mathrm{D}$ & $\mathrm{Fr}$ & $7-12,1-6$ \\
\hline 鳊 Parabramis pekinensis & 0.02 & $14.7-86.7$ & $\mathrm{C}-\mathrm{D}$ & $\mathrm{Fr}$ & $7,8,1,4$ \\
\hline 寡鳞飘鱼 Pseudolaubuca engraulis & 1.08 & $6.6-74.4$ & $\mathrm{C}-\mathrm{D}$ & $\mathrm{Fr}$ & $8-10,12,1,2$ \\
\hline 麦穗鱼 Pseudorasbora parva & 0.13 & $9.4-52.4$ & $\mathrm{C}-\mathrm{D}$ & $\mathrm{Fr}$ & $7-10,12,1,2,4-6$ \\
\hline 银飘鱼 Pseudolaubuca sinensis & 1.78 & $5.9-53.0$ & $\mathrm{~B}-\mathrm{D}$ & $\mathrm{Fr}$ & $7-11,6$ \\
\hline 中华鳑鮍 Rhodeus sinensis & 0.02 & $7.2-30.0$ & $\mathrm{C}-\mathrm{D}$ & $\mathrm{Fr}$ & $8,9,5,6$ \\
\hline 蛇鲍 Saurogobio dabryi & 0.03 & $7.3-12.3$ & $\mathrm{C}$ & $\mathrm{Fr}$ & $7-8,6$ \\
\hline 银鲍 Squalidus argentatus & + & $62.1-64.3$ & $\mathrm{D}$ & $\mathrm{Fr}$ & 12,1 \\
\hline 似鱎 Toxabramis swinhonis & 1.34 & $6.5-82.2$ & $\mathrm{C}-\mathrm{E}$ & $\mathrm{Fr}$ & $7,1,2,6$ \\
\hline 黄尾鲴 Xenocypris davidi & 0.04 & $6.3-9.5$ & $\mathrm{~B}-\mathrm{C}$ & $\mathrm{Fr}$ & 6 \\
\hline 飘鱼属Pseudolaubuca sp. & 0.05 & $8.0-16.3$ & $\mathrm{C}$ & $\mathrm{Fr}$ & 9,6 \\
\hline 鳑鮍亚科 Acheilognathinae sp. & + & 7.4 & $\mathrm{C}$ & $\mathrm{Fr}$ & 6 \\
\hline 鲤科 Cyprinidae spp. & 0.11 & $7.1-21.8$ & $\mathrm{~B}-\mathrm{C}$ & $\mathrm{Fr}$ & $8-10,6$ \\
\hline \multicolumn{6}{|l|}{ 鳅科 Cobitidae } \\
\hline 泥鳅 Misgurnus anguillicaudatus & + & 120.0 & $\mathrm{D}$ & Fr & 11 \\
\hline \multicolumn{6}{|l|}{ 鲿科 Bagridae } \\
\hline 长须黄颡鱼 Pelteobagrus eupogon & + & $57.4-70.3$ & $\mathrm{D}$ & $\mathrm{Fr}$ & 10 \\
\hline 黄颡鱼 Pelteobagrus fulvidraco & 0.01 & $8.6-9.8$ & $\mathrm{~B}$ & $\mathrm{Fr}$ & 6 \\
\hline \multicolumn{6}{|l|}{ 青鳉科 Oryziatidae } \\
\hline 青鳉 Oryzias latipes latipes & 0.01 & $7.6-18.5$ & $\mathrm{~B}-\mathrm{C}$ & $\mathrm{Fr}$ & 7,6 \\
\hline 花鳉科 Poeciliidae & & & & & \\
\hline
\end{tabular}




\begin{tabular}{|c|c|c|c|c|c|}
\hline 种名 Species & $\begin{array}{l}\text { 百分比 } \\
\text { Percentage in } \\
\text { numbers (\%) }\end{array}$ & $\begin{array}{l}\text { 体长范围 } \\
\text { Range of body } \\
\text { length }(\mathrm{mm})\end{array}$ & $\begin{array}{l}\text { 发育阶段 } \\
\text { Developmental } \\
\text { stage }\end{array}$ & $\begin{array}{l}\text { 生态类型 } \\
\text { Ecological } \\
\text { pattern }\end{array}$ & $\begin{array}{l}\text { 出现月份 } \\
\text { Month }\end{array}$ \\
\hline 食蚊鱼 Gambusia affinis affinis & 0.26 & $6.1-35.6$ & $\mathrm{C}-\mathrm{D}$ & $\mathrm{Fr}$ & $7-12,3-6$ \\
\hline \multicolumn{6}{|l|}{ 鱵科 Hemiramphidae } \\
\hline 间下鱵 Hyporhamphus intermedius & 0.16 & $6.6-124.6$ & $\mathrm{C}-\mathrm{D}$ & Es & $7,10,1,5$ \\
\hline \multicolumn{6}{|l|}{ 飞鱼科 Exocoetidae } \\
\hline 尖头燕鳐 Hirundichthys oxycephalus & + & 5.7 & $\mathrm{C}$ & $\mathrm{Ma}$ & 6 \\
\hline \multicolumn{6}{|l|}{ 海龙鱼科 Syngnathidae } \\
\hline 笔状副海龙 Parasyngnathus penicillus & 0.01 & $56.3-58.0$ & $\mathrm{D}$ & $\mathrm{Ma}$ & 9 \\
\hline 尖海龙鱼 Syngnathus acus & 0.01 & $56.2-72.3$ & $\mathrm{D}$ & $\mathrm{Ma}$ & 8 \\
\hline 海马属 Hippocampus sp. & + & 5.0 & $\mathrm{C}$ & $\mathrm{Ma}$ & 6 \\
\hline \multicolumn{6}{|l|}{ 鲻科 Mugilidae } \\
\hline 棱鮻 Liza carinata & 0.03 & $51.8-101$ & $\mathrm{D}$ & Es & 12,1 \\
\hline 鮻 Liza haematocheila & 0.29 & $14.8-90.2$ & $\mathrm{C}-\mathrm{D}$ & Es & $7-11,1,4-6$ \\
\hline 鲻 Mugil cephalus & 0.64 & $16.5-39.4$ & $\mathrm{D}$ & Es & $7-8,3-6$ \\
\hline \multicolumn{6}{|l|}{ 马鲅科 Polynemidae } \\
\hline 四指马鲅 Eleutheronema tetradactylum & 0.28 & $8.4-67.4$ & $\mathrm{C}-\mathrm{D}$ & Es & $7-10,6$ \\
\hline \multicolumn{6}{|l|}{ 鮨科 Serranidae } \\
\hline 中国花鲈 Lateolabrax maculatus & 0.04 & $13.8-42.1$ & $\mathrm{C}-\mathrm{D}$ & Di & $8,4,5$ \\
\hline 鳜 Siniperca chuatsi & 0.55 & $4.0-10.5$ & $\mathrm{~A}-\mathrm{C}$ & $\mathrm{Fr}$ & $7,8,5,6$ \\
\hline \multicolumn{6}{|l|}{ 鱚科 Sillaginidae } \\
\hline 少鳞鱚 Sillago japonica & 0.01 & $11.0-18.4$ & $\mathrm{C}$ & $\mathrm{Ma}$ & 8 \\
\hline 多鳞鱚 Sillago sihama & 0.02 & $11.0-18.2$ & $\mathrm{C}$ & $\mathrm{Ma}$ & 7 \\
\hline \multicolumn{6}{|l|}{ 石首鱼科 Sciaenidae } \\
\hline 棘头梅童鱼 Collichthys lucidus & 0.01 & $5.3-6.8$ & $\mathrm{~A}-\mathrm{B}$ & $\mathrm{Ma}$ & 6 \\
\hline \multicolumn{6}{|l|}{ 银鲈科 Gerreidae } \\
\hline 银鲇科 Gerreidae sp. & 0.01 & $10.1-15.1$ & $\mathrm{C}$ & $\mathrm{Ma}$ & 8 \\
\hline \multicolumn{6}{|l|}{ 鱼刺科 Theraponidae } \\
\hline 细鳞鯻 Terapon jarbua & 0.01 & $12.4-15.9$ & $\mathrm{C}$ & Es & 8,9 \\
\hline \multicolumn{6}{|l|}{ 金钱鱼科 Scatophagidae } \\
\hline 金钱鱼 Scatophagus argus & + & $13.0-31.1$ & $\mathrm{D}$ & Es & 7,8 \\
\hline \multicolumn{6}{|l|}{ 鱼衔科 Callionymidae } \\
\hline 香魦 Repomucenus olidus & 0.13 & $5.0-35.4$ & $\mathrm{C}-\mathrm{D}$ & Es & $8-10,5$ \\
\hline \multicolumn{6}{|l|}{ 塘鳢科 Eleotridae } \\
\hline 尖头塘鳢 Eleotris oxycephala & + & 11.6 & $\mathrm{C}-\mathrm{D}$ & $\mathrm{Fr}$ & 9 \\
\hline 黄䱂 Hypseleotris swinhonis & 0.02 & $10.5-42.3$ & $\mathrm{C}-\mathrm{D}$ & $\mathrm{Fr}$ & 7,5 \\
\hline \multicolumn{6}{|l|}{ 虾虎鱼科 Gobiidae } \\
\hline 长体鯻虾虎鱼 Acanthogobius elongata & 0.01 & $8.3-50.2$ & $\mathrm{D}$ & Es & 6 \\
\hline 黄鯺鯻虾虎鱼 Acanthogobius flavimanus & 0.03 & $6.0-11.6$ & $\mathrm{C}$ & Es & 7,10 \\
\hline 棕鯻虾虎鱼 Acanthogobius luridus & 0.09 & $10.0-58.8$ & $\mathrm{C}-\mathrm{D}$ & Es & $7,8,11,12,2,3,6$ \\
\hline 斑尾鲉虾虎鱼 Acanthogobius ommaturus & 0.15 & $9.3-71.7$ & $\mathrm{C}-\mathrm{D}$ & Es & $7,8,10,5,6$ \\
\hline 普氏细棘虾虎鱼 Acentrogobius pflaumii & 3.12 & $5.3-11.5$ & $\mathrm{~B}-\mathrm{C}$ & Es & $7-10,5,6$ \\
\hline 六丝钝尾虾虎鱼 Amblychaeturichthys hexanema & + & $52.4-57.5$ & $\mathrm{D}$ & Es & 6 \\
\hline 中华钝牙虾虎鱼 Apocryptichthys sericus & 0.01 & $12.8-14.1$ & $\mathrm{C}$ & Es & 8 \\
\hline 阿部鲻虾虎鱼 Mugilogobius abei & 0.04 & $5.5-37.7$ & $\mathrm{C}-\mathrm{D}$ & Es & $7,8,6$ \\
\hline 粘皮鲻虾虎鱼 Mugilogobius myxodermus & 0.02 & $6.9-36.0$ & $\mathrm{C}-\mathrm{D}$ & Es & $9,10,12,5$ \\
\hline 爪哇拟虾虎鱼 Pseudogobius javanicus & + & 30.7 & $\mathrm{D}$ & Es & 5 \\
\hline 波氏吻虾虎鱼 Rhinogobius cliffordpopei & 0.01 & $7.6-18.3$ & $\mathrm{C}-\mathrm{D}$ & $\mathrm{Fr}$ & 9,6 \\
\hline 子棱吻虾虎鱼 Rhinogobius giurinus & 0.50 & $5.5-24.1$ & $\mathrm{C}-\mathrm{D}$ & $\mathrm{Fr}$ & $7-9,5,6$ \\
\hline 髭虾虎鱼 Triaenopogon barbatus & 0.03 & $14.1-16.5$ & $\mathrm{D}$ & Es & $7-9$ \\
\hline 纹缟虾虎鱼 Tridentiger trigonocephalus & 1.28 & $5.7-47.6$ & $\mathrm{C}-\mathrm{D}$ & Es & $7-9,5,6$ \\
\hline 虾虎鱼科 Gobiidae sp. & + & 5.4 & $\mathrm{~B}$ & Es & 5 \\
\hline
\end{tabular}




\begin{tabular}{|c|c|c|c|c|c|}
\hline 种名 Species & $\begin{array}{l}\text { 百分比 } \\
\text { Percentage in } \\
\text { numbers (\%) }\end{array}$ & $\begin{array}{l}\text { 体长范围 } \\
\text { Range of body } \\
\text { length }(\mathrm{mm})\end{array}$ & $\begin{array}{l}\text { 发育阶段 } \\
\text { Developmental } \\
\text { stage } \\
\end{array}$ & $\begin{array}{l}\text { 生态类型 } \\
\text { Ecological } \\
\text { pattern } \\
\end{array}$ & $\begin{array}{l}\text { 出现月份 } \\
\text { Month }\end{array}$ \\
\hline \multicolumn{6}{|l|}{ 弹涂鱼亚科 Periophtalminae } \\
\hline 大弹涂鱼 Boleophthalmus pectinirostris & 0.07 & $12.6-69.4$ & $\mathrm{C}$ & Es & $7-10,2$ \\
\hline 弹涂鱼 Periophthalmus modestus & 1.34 & $6.4-67.2$ & $\mathrm{C}-\mathrm{D}$ & Es & $7-11,4-6$ \\
\hline 青弹涂鱼 Scartelaos histophorus & 0.01 & $29.3-52.8$ & $\mathrm{D}$ & Es & 9,10 \\
\hline \multicolumn{6}{|l|}{ 鳗虾虎鱼亚科 Taenioidinae } \\
\hline 红狼牙虾虎鱼 Odontamblyopus rubicundus & 0.10 & $10.3-153.8$ & $\mathrm{C}-\mathrm{E}$ & Es & $7-9,6$ \\
\hline 须鳗虾虎鱼 Taenioides cirratus & 0.01 & $9.9-13.0$ & $\mathrm{C}$ & Es & 7,9 \\
\hline \multicolumn{6}{|l|}{ 格斗鱼科 Belontiidae } \\
\hline 圆尾斗鱼 Macropodus chinensis & + & $19.2-29.3$ & $\mathrm{D}$ & $\mathrm{Fr}$ & 7,9 \\
\hline \multicolumn{6}{|l|}{ 鳢科 Channidae } \\
\hline 乌鳢 Channa argus & + & 55.9 & $\mathrm{D}$ & $\mathrm{Fr}$ & 7 \\
\hline \multicolumn{6}{|l|}{ 鲬科 Platycephalidae } \\
\hline 鲬 Platycephalus indicus & + & 15.6 & $\mathrm{C}$ & $\mathrm{Ma}$ & 5 \\
\hline \multicolumn{6}{|l|}{ 鳎科 Soleidae } \\
\hline 短吻舌鳎 Cynoglossus abbreviatus & 0.01 & $14.0-63.0$ & $\mathrm{C}-\mathrm{D}$ & $\mathrm{Ma}$ & 5,6 \\
\hline \multicolumn{6}{|l|}{ 鲀科 Tetraodontidae } \\
\hline 双斑多纪鲀 Takifugu bimaculatus & + & 57.2 & $\mathrm{D}$ & Es & 10 \\
\hline 暗纹多纪鲀 Takifugu fasciatus & 0.01 & $33.4-43.9$ & $\mathrm{D}$ & Di & 8 \\
\hline 弓斑多纪鲀 Takifugu ocellatus & 0.10 & $11.5-23.2$ & $\mathrm{D}$ & Es & 7,6 \\
\hline
\end{tabular}

$+:<0.01$ 发育阶段(Developmental stage); A:前弯曲期仔鱼(Preflexion larva); B：弯曲期仔鱼(Flexion larva); C：后弯曲期仔鱼(Postflexion larva); D: 稚鱼(Juvenile); E: 幼鱼(Young fish)。生态类型(Ecological pattern): Ma: 海洋性(Marine fish); Es: 河口性(Estuary fish); Fr: 淡水性(Freshwater fish); Di: 洄游性(Diadromous fish)。

历来是许多重要的经济洄游性鱼类的通道。对于长 江口水域鱼类的物种变动也历来引人关注。Yang et al（1990）在长江口及其近海拖网调查中报道了 53 科 94 种鱼卵、仔稚鱼; Jiang et al (2006) 对长江 口临近海域的鱼卵、仔稚鱼的调查, 共发现 23 科 35 种仔稚鱼。长江口渔场的调查也证实了 40 科 73 种鱼类的存在 ( Li et al, 2004, 2006)。以上的调查 水域主要限制在长江口的临近海区, 对长江口的南 北支入海口的调查较少, 从而出现了以海水性鱼类 为主, 淡水鱼类较少, 近海型种类较多的结果。近 年来, 研究者们加强了沿岸水域的研究, Liu et al (2005) 对崇明北滩的鱼类群落结构分析发现了 19 科 33 种鱼类; Jin et al (2007) 报道了生活在长江 口九段沙盐沼湿地的鱼类约 17 科 30 多种, 且以河 口性的稚鱼为主; Zhang et al (2007) 在冬季对崇 西湿地的调查中插网捕获了以沿岸小型淡水鱼为 主的 7 科 23 种鱼类; 本研究则在长江口碎波带发 现了 31 科共 84 种仔稚鱼类。尽管研究水域、调查 方法和使用的网具不同, 本研究的结果比较充分揭 示了长江口碎波带仔稚鱼具有较高的多样性, 碎波 带存在着丰富的渔业补充资源。比较 Zhong et al (2005) 在 5 个站位点采集到的仔稚鱼, 与本研究
在相同站位点上出现的仔稚鱼在种类组成上具有 一定的相似性，说明了碎波带仔稚鱼的种类组成年 变化以及仔稚鱼利用碎波带作为保育场具有恒定 性。本研究的最优势种也与 Zhong et al (2005, 2007) 一致, 为洄游性的刀鲚, 其数量占全年的 $82.63 \%$ (表 1 ), 在夏季甚至达 $90 \%$ 以上。从而表明, 碎波带是 刀鲚仔稚鱼重要的保育场。而在以往的研究中, 刀 鲚出现的量甚少 (Huang et al, 1989; Zhang et al, 2005 )。本研究的结果相对于刀鲚成鱼的资源衰竭, 形成了极不协调的局面。因此, 必须通过整治长江 口通道中的有害网具, 保持河海通道的畅通, 以确 保碎波带水域保育场的刀鲚仔鱼顺利进入海洋生 长。对于如何维持刀鲚的补充资源量, 恢复刀鲚历 史上的资源量，还有待于从资源保护和管理等多方 面进行进一步探讨。

周年出现的种类以太湖新银鱼、䱗、麦穗鱼等淡水 性鱼类为主，而大多数的河口性、海洋性及洄游性 鱼类仔稚鱼在碎波带仅呈现季节性出现或短时出 现, 特别是温度较高的春、夏季出现种类较多（表 1), 该现象可能与鱼类的繁殖季节以及适温有着一 定的关系 (图 4)。在碎波带出现的河口性鱼类和淡 水性鱼类, 不仅种类丰富, 且数量大, 而海洋性鱼 
类虽然种类较多，但是数量却相对较少。由此可见 仔稚鱼将长江口沿岸碎波带作为其重要栖息地和 保育场也因物种而异，并具有季节选择性。

\section{2 环境因子对仔稚鱼丰度和分布的影响}

仔稚鱼的种类数、个体数和丰度的月变化趋势 与碎波带的水温的月变化趋势基本一致（图 2，4）, 可见水温的变化影响着仔稚鱼的种类出现和数量 变动。盐度的变化也同样影响着仔稚鱼的分布。长 江口南支是江水主要入海通道, 其径流量导致了各 站位平均盐度相对较低, 而北支的上游 (Sts.10-11) 主要受长江冲淡水的影响, 因此南支和北支上游碎 波带所出现的种类主要是淡水性鱼类和洄游性鱼 类。长江口的北支的下游 (Sts.12-13) 受潮汐的 影响较大, 平均盐度较高, 而且, 近几年随着咸潮 对北支影响的加剧 (Song et al, 2002; Wu et al, 2006)，北支下游碎波带出现的种类主要以海洋性 和河口性鱼类为主。同样，位于南汇浅滩的 Sts.1

\section{参考文献:}

Bregje B, Kris H, Jan M. 2001. Factors influencing fish and macrocrustacean communities in the surf zone of sandy beaches in Belgium: Temporal variation [J]. Journal of Sea Research, 46: 281-294.

Doherty P, Mcilwain J. 1996. Monitoring larval fluxes through the surf zone of Australian coral reefs [J]. Marine and Freshwater Research, 47: 383-390.

Fujita S, Kinoshita I, Takahashi I, Azuma K. 2002. Species composition and seasonal occurrence of fish larvae and juveniles in the Shimanto Estuary, Japan [J]. Fisheries Science, 68(2): 364-370.

Huang JB, Zhang GX, Zhang XS, Li GB. 1992. The distribution of larva of Hemisalanx prognathus in the mouth of the Yangtze River and its main morphological characteristics [J]. Marine Fisheries, 1:17-10. [黄晋彪, 张国祥, 张雪生, 李根宝. 1992. 长江口前领间银鱼仔鱼的分布及其 主要形态特征. 海洋渔业, 1:17-10.]

Huang JB, Zhang XS. 1989. Analysis on the resource of Coilia nasus in the Yangtze River estuary [J]. Fisheries Sicence \& Technology Information, 16(6): 173-175. [黄晋彪, 张雪生. 1989. 长江口刀魰资源试析. 水产 科技情报, 16(6): 173-175.]

Jiang M, Shen XQ. 2006. Abundance distributions of pelagic fish eggs and larva in the Changjiang River estuary and vicinity waters in summer [J]. Marine Sciences, 30 (6): 92-97. [蒋 玫, 沈新强. 2006. 长江口及邻 近水域夏季鱼卵、仔鱼数量分布特征. 海洋科学, 30 (6): 92-97.]

Jiang M, Shen XQ, Chen LF. 2006. Relationship between with abundance distribution of fish eggs, larvae and environmental factors in the Changjiang Estuary and vicinity waters in spring [J]. Marine Environmental Science, 26 (2): 37-44. [蒋 玫, 沈新强, 陈莲芳. 2006. 长江口及邻近水域春季鱼卵仔鱼与环境因子的关系. 海洋环境科学, 26 (2): 37-44.]

Jin BS, Fu CZ, Zhong JS, Li B, Chen JK, Wu JH. 2007. Fish utilization of a salt marsh intertidal creek in the Yangtze River estuary, China [J]. Estuarine, Coastal and Shelf Science, 73 (3-4): 844-852.

Jin BS, Fu CZ, Zhong JS, Chen JK, Wu JH. 2007. Effects of fyke net mesh size and sampling period on the catch of nekton in an intertidal creek [J].
-3 受海水的影响也较大, 平均盐度较高, 出现的 种类与北支下游碎波带有相同的趋势。

碎波带仔稚鱼类的多样性指数结果表明了仔稚 鱼种类和数量均很高, 存在着一定的资源补充量。 因此要维持和提高长江口渔业资源量, 在严格执行 禁渔、休渔政策的同时，必须加强对碎波带环境的 监测和保护，严禁污染的乱排放，做到既保护长江 口的生态环境，又保障渔民的利益，保证长江口水 域渔业资源的可持续利用。

致谢: 在本研究的野外标本采集、室内实验、 数据整理过程中, 得到了浙江省淡水水产研究所练 青平, 上海海洋大学生命学院硕士研究生李黎、刘 否、林楠、吴美琴、葛珂珂, 复旦大学博士研究生 金斌松，华东师范大学博士张衡等的大力协助，并 提出宝贵意见，在此一并致谢!

Acta Hydrobiology Sinica, 31(1): 39-44

Kendall AWJ, Ahlstrom EH, Moser HG. 1984. Early life history stages of fishes and their characters [A]. In: Ontogeny and Systematics of Fishes [M]. Lawrence: The American Society of Ichthyologists Herpetologists, Spec Publ.

Kinoshita I. 1993. Ecological study on larvae and juveniles of sparine fishes occurring in surf zones of sandy beaches [J]. Bulletin of Marine Sciences and Fisheries, Kochi University, 13: 21-99. (in Japanese with English abstract)

Lasiak TA. 1981. Nursery grounds of juveniles teleosts: Evidence from the King's Beach, Port Elizabeth [J]. South African Journal of Science, 77: 388-390.

Li JS, Li SF, Cheng JH. 2006. The composition and diversity of fishes on fishing grounds of Changjiang estuary[J]. Marine Fisheries, 28 (6): 37-41. [李建生, 李圣法, 程家骅. 2006. 长江口渔场鱼类组成和多样 性. 海洋渔业, 28 (6): 37-41.]

Li JS, Li SF, Ren YP, Cheng JH. 2004. Seasonal variety of fishery biology community structure in fishing ground of the Yangtze estuary [J]. Journal of Fishery Sciences of China, 11 (53): 432-439. [李建生, 李圣 法, 任一平, 程家骅. 2004. 长江口渔场渔业生物群落结构的季节变 化. 中国水产科学, 11 (53): 432-439.]

Liu K, Xu DP, Zhang MY, Duan JR, Shi WG. 2005. Preliminary studies on biodiversity of fish community on north beach of Chongming island [J]. Resources and Environment in the Yangtze Basin, 14(4): 418-421. [刘 凯, 徐东坡, 张敏莹, 段金荣, 施炜纲. 2005. 崇明北滩鱼类群落生 物多样性初探. 长江流域资源与环境, 14(4): 418-421.]

Ma KP. 1994. Measure methods of biodiversity [A]. In: Biology Diversity Research Principles and Methods [M]. Beijing: China Science and Technology Press. [马克平. 1994. 生物多样性的测度方法. 见: 钱迎 倩, 马克平. 生物多样性研究的原理和方法. 北京: 中国科学技术 出版社.]

Ruple DL. 1984. Occurrence of larval fishes in the surf zone of a northern Gulf of Mexico barrier island [J]. Estuarine, Coastal and Shelf Science, 
18: 191-208.

Senta T, Kinoshita I. 1985. Larval and juvenile fishes occurring in surf zones of western Japan [J]. Transactions of the American Fisheries Society, 114: 609-618.

Shan XJ, Xian WW, Wu YF. 2005. Dynamic changes in the ichthyoplankton community structure after the sluice of the Three-Gorges Dam[J]. Journal of Ocean University of Qingdao, 35(6): 936-940. [单秀娟, 线 薇薇, 武云飞. 2005. 三峡工程蓄水前后秋季长江口鱼类浮游生物群 落结构的动态变化初探. 中国海洋大学学报, 35(6): 936-940.]

Song ZY, Mao LH. 2002. Salt water encroachment at the Yangtze River Estuary [J]. Water Resources Protection, 3: 27-30. [宋志尧, 茅丽华. 2002. 长江口盐水入侵研究. 水资源保护, 3: 27-30.]

Tang WQ, Zhu TJ, Chen JK, Han HF, Sun Y. 2003. Resources and conservation valuation of fishes of Jiuduansha wetland in Changjiang River estuary [J]. Journal of Shanchai Fisheries University, 12 (3): 193-200. [唐文乔, 诸廷俊, 陈家宽, 韩洪发, 孙 瑛. 2003. 长江口 九段沙湿地的鱼类资源及其保护价值. 上海水产大学学报, 12 (3): 193-200.]

Tang YM. 1983. Oceanography [M]. Beijing: China Agricultural Press. [唐 逸民. 1983. 海洋学. 北京: 中国农业出版社.]

Wang YH, NiY. 1983. The fisheries resource and present condition of the Yangtze River estuary in Shanghai[J]. Fisheries Sicence \& Technology Information, 2: 6-9. [王幼槐, 倪 勇. 1983. 上海市长江口区的渔业 资源和渔业现状. 水产科技情报, 2: 6-9.]

Wu GZ. 1989. The ecological characteristics of distribution of eggs, larvae and juveniles of the Engeraulis japonicus (Temminck \& Schlegel) and Anchoviella commersonii (Leceped) in the Changjiang River estuary [J]. Oceanologia Et Limnologia Sinica, 20 (3): 217-229. [吴光宗. 1989. 长 江口海区的鳀鱼和康氏小公鱼的鱼卵和仔稚鱼的生态特征. 海洋与 湖沼, 20 (3): 217-229.]

Wu HL, Shao GZ, Lai CF. 1999. Latin-Chinese Dictionary of Fishes Names [M]. Keelung: Sueichan Press. [伍汉霖, 邵广昭, 赖春福. 1999. 拉汉 世界鱼类名典. 基隆: 水产出版社.]

Wu JW, Liu XC, Pan LH. 2006. Hydrodynamic simulation of control project on saltwater intrusion from north branch of Yangtze River Estuary [J]. Advances in Science and Technology of Water Resources, 26(4): 43-55. [吴继伟, 刘新成, 潘丽红. 2006. 长江口北支咸潮倒灌控制工程水 动力数值模拟. 水利水电科技进展, 26(4): 43-55.]

Xu ZL, Yuan Q, Jiang M, Zang ZJ. 1999. An investigation of fish eggs, larvae and juveniles in the Changjiang estuary [J]. Journal of Fishery Sciences of China, 6(5): 63-64. [徐兆礼, 袁 騏, 蒋 玫, 藏增加. 1999. 长江口鱼卵和仔、稚鱼的初步调查. 中国水产科学, 6(5): 63-64. ]
Yang DL, Wu GZ, Sun JR. 1990. The investigation of pelagic eggs, larvae and juveniles of fishes at the mouth of the Changjiang River and adjacent areas [J]. Oceanologia et Limnologia Sinica, 21 (4): 346-355. [杨东莱，吴光宗，孙继仁. 1990. 长江口及其邻近海区的浮性鱼卵 和仔稚鱼的生态研究. 海洋与湖沼, 21(4): 346-355.]

Zhang H, He WS, Tong CF, Lu JJ. 2007. Species composition and biodiversity of the intertidal fishes in Chongxi wetland in winter in Changiiang estuary [J]. Resources and Environment in the Yangtze Basin, 16(3): 308-313. [张 衡, 何文珊, 童春富, 陆健健. 2007. 崇 西湿地冬季潮滩鱼类种类组成及多样性分析. 长江流域资源与环境, 16(3): 308-313.]

Zhang MY, Xu DP, Liu K, Shi WG. 2005. Studies on biological characteristic and change of resource of Coilia nasus Schlegel in the lower reaches of the Yangtze River [J]. Resources and Environment in the Yangtze Basin, 14(6): 694-698. [张敏莹, 徐东坡, 刘 凯, 施炜纲. 2005. 长江下游刀鲚生物学及最大持续产量研究. 长江流域资源与 环境, 14(6): 694-698.]

Zhong JS. 2006. Study on immigration mechanism of fish larvae into the inlet as their nurseries [J]. Bulletins of Marine Sciences and Fisheries Kochi University, 24:71-137(in Japanese with English abstract).

Zhong JS, Fu CZ, Yu WW, Wu MQ, Zhang ZP, Gong XL, Hu F. 2006. Occurrence and stay period of the juvenile Liza haematocheila in the surf zone of Yangtze Estuary [J]. Journal of Shanchai Fisheries University, 15(3): 318-323. [钟俊生, 傅萃长, 郁蔚文, 吴美琴, 张增 频, 龚小玲, 胡 芬. 2006. 鮻鱼稚鱼在沿岸碎波带的出现和滞留时 间. 上海水产大学学报, 15(3): 318-323.]

Zhong JS, Yu WW, Liu BL, Gong XL, Bo HJ, Hu F, Ding FY. 2005. Seasonal occurrences of fish larvae and juveniles in the surf zone of the Yangtze River estuary[J]. Journal of Shanghai Fisheries University, 14(4): 375-382. [钟俊生, 郁蔚文, 刘必林, 龚小玲, 薄欢军, 胡 芬, 丁峰元. 2005. 长江口沿岸碎波带仔稚鱼种类组成和季节性变化. 上 海水产大学学报, 14(4): 375-382.]

Zhong JS, Wu MQ, Lian QP. 2007. Composition of fish larvae and juveniles in spring and summer in surf zone of Yangtze River estuary [J]. Journal of Fishery Sciences of China, 4(3): 436-443. [钟俊生, 吴美琴, 练青平. 2007. 春夏季长江口沿岸碎波带仔稚鱼的种类组成. 中国水产科学, 4(3): 436-443.]

Zhu XH, Liu D, Sha XK. 2002. Relationship between community structure of ichthyoplankton and environmental factors in the estuary of Changjiang River in spring [J]. Studia Marina Sinica, 44: 169-179. [朱 金华, 刘 栋, 沙学坤. 2002. 长江口春季鱼类浮游生物群落结构与 环境因子的关系. 海洋科学集刊, 44: 169-179.] 